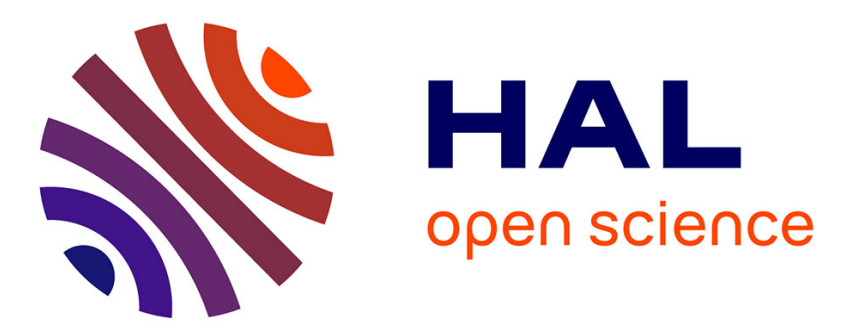

\title{
A Bayesian Packet Sharing Approach for Noisy IoT Scenarios
}

\author{
Anna Maria Vegni, Valeria Loscrì, Alessandro Neri, Marco Leo
}

\section{To cite this version:}

Anna Maria Vegni, Valeria Loscrì, Alessandro Neri, Marco Leo. A Bayesian Packet Sharing Approach for Noisy IoT Scenarios. 1st International Workshop on Interoperability, Integration, and Interconnection of Internet of Things Systems (I4T 2016), Apr 2016, Berlin Germany. hal-01262024

\section{HAL Id: hal-01262024 \\ https://hal.inria.fr/hal-01262024}

Submitted on 26 Jan 2016

HAL is a multi-disciplinary open access archive for the deposit and dissemination of scientific research documents, whether they are published or not. The documents may come from teaching and research institutions in France or abroad, or from public or private research centers.
L'archive ouverte pluridisciplinaire HAL, est destinée au dépôt et à la diffusion de documents scientifiques de niveau recherche, publiés ou non, émanant des établissements d'enseignement et de recherche français ou étrangers, des laboratoires publics ou privés. 


\title{
A Bayesian Packet Sharing Approach for Noisy IoT Scenarios
}

\author{
Anna Maria Vegni*, Valeria Loscrí ${ }^{\dagger}$, Alessandro Neri*, and Marco Leo* \\ * Dept. of Engineering, Roma Tre University \\ COMLAB Telecommunication Laboratory, Rome, Italy \\ Email:\{amvegni,neri,marco.leo\}@uniroma3.it \\ ${ }^{\dagger}$ Inria Lille - Nord Europe, Lille, France \\ Email: valeria.loscri@inria.fr
}

\begin{abstract}
Cloud computing and Internet of Things (IoT) represent two different technologies that are massively being adopted in our daily life, playing a fundamental role in the future Internet. One important challenge that need to be handled is the enormous amount of data generated by sensing devices, that make the control of sending useless data very important. In order to face with this challenge, there is a increasing interest about predictive approaches to avoid to send high spatio-temporal correlated data. Belief Propagation (BP) algorithm is a method of performing approximate inference on arbitrary graphical models that is becoming increasingly popular in the context of IoT. By exploiting BP, we can derive effective methods to drastically reduce the number of transmitted messages, while keeping high the data throughput in the global information system.
\end{abstract}

In this paper, we propose a BP approach in a hierarchical architecture with simple nodes, gateways and data centers. We evaluate the error bounding and propose a corrective mechanism to keep a certain quality of the global information in the architecture considered.

Keywords-IoT-based, Belief Propagation, Cloud.

\section{INTRODUCTION}

Recently, with the evolution of the Internet and related technologies, there has been an evolution of a new emerging paradigm, namely the Internet of Things (IoT) [2]. In IoT scenarios, a large number of devices -and more in general objects- are seamlessly connected to each another for information sharing through the Internet. All these devices connected to the IoT may be of heterogeneous types with respect to their operational mode, and communication technologies. As one of the main strengths behind the IoT paradigm, it is the high impact on several aspects of everyday-life, from working to the domestic fields. As an instance, domotics [13], e-health [7], and smart cities [4], [9] are main application scenarios where the IoT paradigm is expected to play a leading role in the next future.

From the above considerations, and due to the huge amount of heterogeneous devices, information sharing among IoT devices is one of the biggest challenges. Classic Internet approaches need to be revised to address the complex requirements imposed by IoT. This asks for the development of intelligent algorithms for routing [1], information sharing security [6], [5], novel network paradigms [8], [14], [16], new services [11], [15], and advanced techniques for data fusion [3].
A few related works have addressed the issue of forwarding data among IoT devices, by modelling the IoT network as a Bayesian network [3], [10], [12]. Under this hypothesis, Bijarbooneh et al. [3] present an adaptive sensing belief propagation algorithm, where each node updates its belief about the environment status by incorporating its local measurement with the beliefs of its neighboring nodes and the belief obtained in the past. Finally, a cloud-based network architecture allows reducing energy consumption for data fusion and storage tasks through a carefull selection of the set of IoT nodes involved in the distributed estimate of the environment status.

In this paper, we address the connectivity issue among IoT heterogeneous devices for data sharing, under the hypothesis of Bayesian network i.e. . We assume each IoT device represents a node in the IoT network with some sensing and processing capabilities. Moreover, these devices may be located at different places across the globe. They are connected to the Internet, althgough the rate of data transfer, and the supported security level may be different. Each node needs to get information about its local environment, in order to perform some task and/or to provide this information to a higher decision level. As an instance, an IoT node deployed in a domestic network may need information about current and future usage of some limited resources, like energy or communication bandwidth, to orchestrate their comsumption with the help of the opther IoT devices controlling specific appliances. Data exchange among devices allows a single node to increase its own knowledge of global information and optimize the scheduling of the tasks that it has to accomplish with the usage of a shared resource.

In this paper, we present a data sharing approach based on Pearl's Belief Propagation (BP) algorithm in the IoT context with a cloud-based architecture. BP is an iterative technique mainly used for solving inference problems. In the IoT context, the belief of a device (e.g., a sensor node) is the data measurement. The BP infers the measurements of other neighboring devices, especially in cases where the data is missing. Moreover, the BP technique allows to correct the errors that can occur in the data propagation. At each run, the BP provides to the devices both spatial and temporal cooperation. Indeed, in BP-based approaches, each sensor node determines its belief by incorporating its local measurement with the beliefs of its neighboring nodes, as well as its beliefs obtained in the past run. Then, at each run, we assume a node is able to $(i)$ reduce the own distortion level (i.e., estimation error on global information), and (ii) provide an update of the 


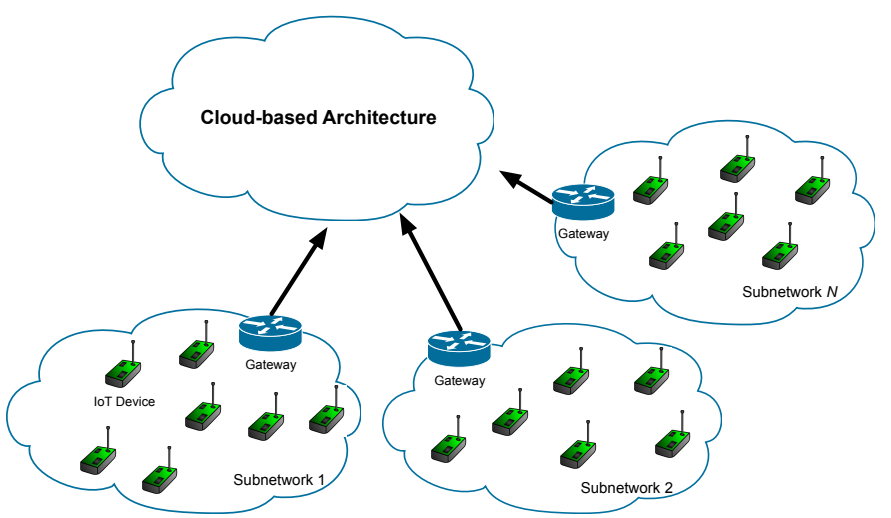

Figure 1: IoT network model with several subnetworks. The main entities are: IoT devices, equipped with sensing instrumentation, that selectively send data to the gateway and implement the BP algorithm, the gateways, and the cloud.

global information to be shared with other neighboring nodes.

This paper is organized as follows. Section II describes the reference cloud-based architecture for the IoT scenario. Due to the heterogeneity of the IoT devices, we assume a multinetwork scenario with a plethora di interconnected devices. In Section III we present our technique based on BP algorithm, for data sharing in a noisy IoT scenario. The presence of errors along the message reconstruction phase occurring at each receiver node can be mitigate through our BP's algorithm. Finally, conclusions are drawn at the end of this paper.

\section{NETWORK MODEL}

In our architecture, we consider different entities with specific computational and communication capabilities and functionalities. As illustrated in Figure 1 the IoT network model may comprise several sub-networks (i.e., from 1 to $N$ ), associated with different applications. Indeed, due to the huge amount of IoT devices, we assume each sub-network is composed by IoT devices connected to each others for data sharing, and a gateway that interacts with the "external world". The role of the gateways consists into relaying the messages to the cloud, which is responsible of typical cloud-based services (e.g., data fusion, storage, etc.).

Each device performs sensing and processing activities. Our network model supports multi-hop routing, and the gateways collect data and forward them to the cloud. To support high scalability of the architecture, gateways implement publish-subscribe message passing mechanisms based on message brokers. Depending on the device capabilities, publishsubscribe mechanisms can be supported even at IoT node level. In this scenario, we assume that each IoT node can be in two states, either idle or active. A node is in a idle state, when it disconnects its radio, and it cannot send and receive data. On the other hand, a node is in active state when it can perform sensing activities, and can send and receive messages.

Moreover presence of errors in the connectivity links among IoT devices has to be accounted for. This can affect communication reliability, and also cause packet losses. Packet retransmission are then necessary to overcome packet errors

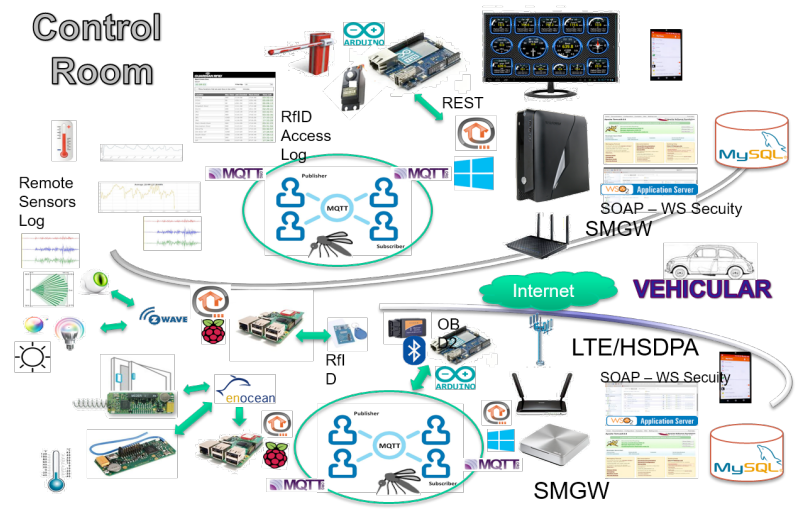

Figure 2: DAHMS's proof of concept architecture

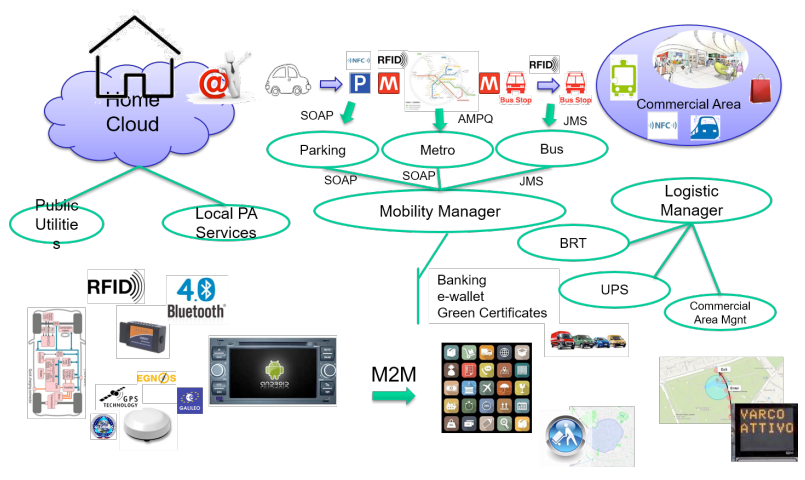

Figure 3: LOGON's architecture

and losses. At the same time, the increase of message retransmissions affects energy consumption on each IoT devices, latency in information sharing, and in the extreme case, network congestion.

In order to keep low the effective number of retransmissions, each active sensor implements an error conceiling strategy based on a BP message passing algorithm with a twofold objective i.e., (i) to recover missing data through the BP algorithm, and (ii) to reconstruct "incomplete" or "corrupted" messages.

The proposed architecture has been adopted by the authors in the design of the proof of concept of DAHMS and LOGON projects, both funded by the Italian Ministery of Economic Development in the framework "New Technologies for the Made in Italy", carried out at the Radiolabs research center labs. DAHMS (Distributed Architecture Home Modular Multifunctional Systems) scope is the improvement of the quality of life and the degree of self-sufficiency of chronically ill and elderly and disabled persons through the integration of Home Automation and remote heathcare functionalities. LogOn (Logistic Open Network) concerns the goods logistic in historical art cities with high level of tourism economy.

Figure 2 and Figure 3 depict the set of technologies and communication systems that are part of the DAHMS and LOGON proofs of concept. 
The Secure Mediation GateWay (SMGW) represents the conjunction element among the devices within the Control Room and on-board (vehicular) devices.

The on-board devices are able to send events and receive commands from a set of control interfaces accessible both from the Control Room, the vehicles, and also from mobile devices (Android or iOS).

The information messages sent from each device follow a publish/subscribe framework able to forward MQTT (MQ Telemetry Transport) messages. MQTT is a messaging protocol working on the top of TCP/IP, that has been projected for specific situations where low impact and limited bandwidth are required. All the devices are equipped with MQTT and will be in charge to forward informations about events or commands. Through the federated system of SMGW, the informations published via MQTT will be available in a seamless way from each intraSMGW domain, as described in the architectural scheme of SMGW. Within each domain, the devices Raspberry or Arduino equipped- are in charge of exchanging event/commands and messages with the SMGW. Also, the SMGW exports each message in a secure way towards all the other SMGWs.

\section{A BP TECHNIQUE FOR ERROR CORRECTION}

In this section, we investigate the proposed BP technique in IoT noisy scenarios. Our aim is to reduce the message errors through an iterative algorithm that corrects and updates the received data at each run. With regards to Figure 1, each device is initiated in active mode, and transmits messages to its own neighbors. A message is related to local data measurements, sampled at a fixed time step. The global information, related to a given sub-network, is then obtained from the contributions coming from each IoT device within the subnetwork.

Let us assume that the distributed sensing system consists of $N$ IoT nodes, interconnected in various ways. Each IoT node collects a set of data provided by several sensors. Our scope is then to estimate the state $\mathbf{X}$ of the sensed environment starting from the sets $\left\{\mathbf{D}_{i}\right\}$ of data collected by the individual nodes related to non overlapping regions $\left\{\mathbf{R}_{i}\right\}$. Here $\mathbf{X}$ is modeled as a dynamical Random Field. More in detail we focus our attention to the case in which the dynamical (i.e. temporal) behaviour of the system can be described by a linear model, while the spatial behavior is described by a Markov Random Field.

We incidentally recall that given a finite rectangular lattice $L=\left\{(i, j), \quad 1 \leq i \leq N_{1}, \quad 1 \leq j \leq N_{2}\right\}$, a neighborhood system associated with $L$ is, by definition, a collection of subsets $\eta=\left\{\eta_{i j}\right\}$ with the property that each subset $\eta_{i j}$, namely the neighborhood of $i, j$, is such that:

$$
\begin{array}{ll}
\text { - } & (i, j) \in \eta_{i j}, \\
\text { - } & \text { if }(k, l) \in \eta_{i j}, \text { then }(i, j) \in \eta_{k l}, \quad \forall(i, j) \in L
\end{array}
$$

It follows that a random field $\mathbf{X}$ is said to be a Markov Random Field w.r.t. $(L, \eta)$ if and only if:

$$
\begin{aligned}
& P(X(i, j) / X(k, l),(k, l) \in L-\{(i, j)\})= \\
& =P\left(X(i, j) / X(k, l),(k, l) \in \eta_{i j}-\{(i, j)\}\right), \\
& \forall(i, j) \in L .
\end{aligned}
$$

Then, in the distributed estimation scheme we can take advantage of the Hammersley-Clifford Theorem, stating that the joint distribution of a Markov Random Field w.r.t. $(L, \eta)$ is of the form

$$
P_{\mathbf{X}}(\mathbf{x})=\frac{1}{Z} \exp \{-U(\mathbf{x})\},
$$

where $Z$ is a normalizing constant and

$$
U(\mathbf{x})=\sum_{\forall \text { clique } c} V_{c}(\mathbf{x}),
$$

is the energy function, and $V_{c}(\mathbf{x})$ is the potential associated with clique $c \in C$.

The only constraint on the clique potential $V_{c}(\mathbf{x})$ is that it depends only on the restriction of $\mathrm{x}$ to $C$. Nevertheless, here we focus our attention on those Markov Random Fields for which the potential function consists only of a set of singleton potentials, defined on single variables, and on a set of pairwise potentials, defined on pairs of variable.

To derive the distributed state estimation model, here we resort to the unified representation for both Bayesian Networks and Random Markov Fields, constituted by the Factor Graphs. Factor Graphs use factor nodes to describe the factorization property of the joint distribution, as the one stated by the Hammersley-Clifford Theorem.

At this aim, for sake of compactness of the notation, without loss of generality, we assume that the sensor data $\mathbf{D}_{i}$ and $\mathbf{D}_{j}$ provided by the sensors respectively connected to the $i$-th and $j$-th node cover two non-overlaping areas, and that the overall state space $\mathcal{X}$ is the Cartesian product of the state subspaces $\mathcal{X}_{i}$ associated to the environmental variables related to the areas covered by the individual nodes.

By associating each node $i$ of a sub-network, with a random variable $\mathbf{X}_{i}$ that represents the local information, and by considering a set of edges $E$, we can write the joint distribution as:

$$
P_{\mathbf{X}}(\mathbf{x})=\prod_{i} \psi_{i}\left(\mathbf{x}_{i}\right) \prod_{(i, j) \in E} \psi_{i j}\left(\mathbf{x}_{i}, \mathbf{x}_{j}\right),
$$

where the function $\psi_{i j}()$ represents the message exchange among node $i$ and $j$. In practice, $p\left(\mathbf{x}_{i}\right)$ represents the marginal distribution of $i$-th node, and the BP allows the computation of the marginal distribution at each node $i$.

From rate-distortion theory, given a one-dimensional random variable $\widehat{X}(X)$ is the representation of $X$, so that

$$
\widehat{X} \in\left\{1,2, \ldots, 2^{n R}\right\},
$$

where $R$ are the bits needed for the representation of $X$.

Then, the distortion function is a mapping

$$
d: \mathcal{X} \times \widehat{\mathcal{X}} \rightarrow \mathbb{R}^{+},
$$

from the set of source alphabet pairs $\mathcal{X}$ into the set of nonnegative real numbers. It measures the cost of representing symbol $x$ by $\hat{x}$. Each node has the aim to minimize the own distortion level. A distortion measure is said to be bounded if the maximum value of the distortion is finite i.e.,

$$
d_{\max }:=\max _{x \in \mathcal{X}, \widehat{x} \in \mathcal{X}} d(x, \widehat{x})<\infty .
$$


For the distortion, we can assume a squared-error definition i.e.,

$$
d(x, \widehat{x})=(x-\widehat{x})^{T}(x-\widehat{x}),
$$

from which we can derive the distortion between sequences $x^{n}$ and $\hat{x}^{n}$ as

$$
d\left(x^{n}, \hat{x}^{n}\right)=\frac{1}{n} \sum_{i=1}^{n} d\left(x_{i}, \hat{x}_{i}\right)
$$

It follows that the distortion associated with a $\left(2^{n R}, n\right)$ code is defined as:

$$
D=E\left[d\left(X^{n}, g_{n}\left(f_{n}\left(X^{n}\right)\right)\right)\right],
$$

where $f_{n}: \mathcal{X}^{n} \rightarrow\left\{1,2, \ldots, 2^{n R}\right\}$, and $g_{n}:\left\{1,2, \ldots, 2^{n R}\right\} \rightarrow$ $\hat{\mathcal{X}}^{n}$.

Finally, we can derive the information rate distortion function $R(D)$ for a source $X$ with distortion measure $d(x, \hat{x})$ as:

$$
R(D)=\min I(X ; \hat{X})
$$

where $I(X ; \hat{X})$ is the mutual information. Notice that Eq. (10) is subject to the following constraint

$$
p(\hat{x} \mid x): \sum_{(x, \hat{x})} p(x) p(\hat{x} \mid x) d(x, \hat{x}) \leq D,
$$

that is, the minimization of the mutual information is over all conditional distribution $p(\hat{x} \mid x)$ for which the jointly distribution $p(x, \hat{x})$ satisfies the expected distortion constraint.

From (9), the expectation value respect to the probability distribution on $\mathcal{X}$ is as follows:

$$
D=\sum x^{n} p\left(x^{n}\right) d\left(x^{n}, g_{n}\left(f_{n}\left(x^{n}\right)\right)\right) .
$$

Now, in order to solve previous equation, we need the estimation of $x_{i}$. This can be provided through the BP algorithm. This is a message passing algorithm for the calculation of $a$ posteriori probabilities of nodes of a loop-free Factor Graph, given a priori probabilities and observations. As known, the $\mathrm{BP}$ algorithm is a graphical model to represent conditional independence relations of large numbers of random variables.

Since the BP algorithm is a message-passing technique between nodes, and represents an update to the outgoing message from $i$-th node to $j$-th neighboring node, we can state that the message from $i$-th to $j$-th node related to the local information $\mathbf{x}_{i}$ is proportional to

$$
m_{j i}\left(\mathbf{x}_{i}\right) \propto \int \psi_{j i}\left(\mathbf{x}_{j}, \mathbf{x}_{i}\right) \psi_{j}\left(\mathbf{x}_{j}\right) \prod_{u \in \Gamma_{j} \backslash i} m_{u j}\left(\mathbf{x}_{j}\right) d \mathbf{x}_{j}
$$

where the incoming messages from previous iteration are represented by $m_{u j}$. This equation represents the message update operation that is performed in the BP's algorithm.

Notice that the BP is capable to compute the exact marginalization in the case of tree-structured graphical models, and this means that (13) converges in a finite number of iterations, limited to a superior bound, that is the length of the longest path in the graph.

The BP algorithm starts with a "belief updating" phase, where the a posteriori probabilities of the random variable

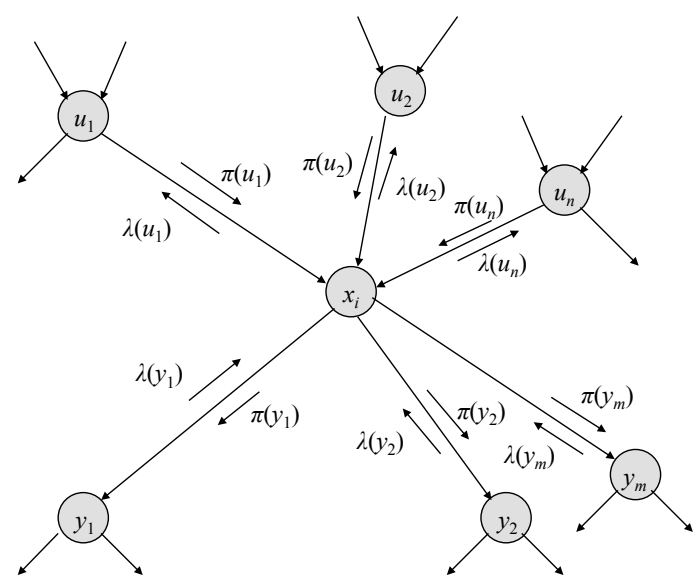

Figure 4: Architecture of the IoT Bayesian network as a graph, for the computation of the BP algorithm.

$\mathbf{x}_{i}$ associated to the $i$-th node, i.e. $B E L\left(\mathbf{x}_{i}\right)$, is computed through the information about the evidence coming from the neighboring nodes i.e., $B E L\left(\mathbf{x}_{i}\right)=\alpha \mu\left(\mathbf{x}_{i}\right)$, where $\mu\left(\mathbf{x}_{i}\right)$ represents the double contribution from "child" and "parent" nodes w.r.t. the $i$-th node i.e.,

$$
\mu\left(\mathbf{x}_{i}\right)=\lambda\left(\mathbf{x}_{i}\right) \pi\left(\mathbf{x}_{i}\right)
$$

with

$$
\begin{gathered}
\lambda\left(\mathbf{x}_{i}\right)=\prod_{j} \lambda_{\mathbf{x}_{j}}\left(\mathbf{x}_{i}\right) \\
\pi\left(\mathbf{x}_{i}\right)=\sum_{\mathbf{u}_{1}, \ldots, \mathbf{u}_{n}} P\left({ }_{i} \mid \mathbf{u}_{1}, \ldots, \mathbf{u}_{n}\right) \prod_{k} \pi_{\mathbf{x}_{i}}\left(\mathbf{x}_{k}\right),
\end{gathered}
$$

where $j$ and $k$ are the indexes for the child and parent nodes, respectively. Figure 4 depicts a schematic of an IoT Factor Graph, assumed as a graph with parent and child nodes with respect to the $\mathbf{x}_{i}$ node. The computation of the a posteriori probability of the node $\mathbf{x}_{i}$, given all evidence except for the information coming from the $j$-th child node, is obtained through the parent-to-child message for the child node whose information is excluded. The message from the $i$-th parent node $\mathbf{x}_{i}$ to the $j$-th child node $\mathbf{y}_{j}$ is denoted as $\pi_{\mathbf{y}_{j}}\left(\mathbf{x}_{i}\right)$, whose expression is:

$$
\pi_{\mathbf{y}_{j}}\left(\mathbf{x}_{i}\right)=\alpha \prod_{m \neq j} \lambda_{\mathbf{y}_{m}}\left(\mathbf{x}_{i}\right) \sum_{\mathbf{u}_{1}, \ldots, \mathbf{u}_{n}} P\left(\mathbf{x}_{i} \mid \mathbf{u}_{1}, \ldots, \mathbf{u}_{n}\right) \prod_{k} \pi_{\mathbf{x}_{i}}\left(\mathbf{u}_{k}\right) .
$$

Finally, the computation of the conditional probability of the evidence coming from the children of $\mathbf{x}_{i}$ given different possible values for the random variable corresponding to the $i$-th node is obtained through the message exchange from the $j$-th child node to the $k$-th parent node as:

$$
\lambda_{\mathbf{x}_{i}}\left(\mathbf{u}_{i}\right)=\beta \sum_{\mathbf{x}_{i}} \lambda\left(\mathbf{x}_{i}\right) \sum_{\mathbf{u}_{k} ; k \neq i} P\left(\mathbf{x}_{i} \mid \mathbf{u}_{1}, \ldots, \mathbf{u}_{n}\right) \prod_{k \neq i} \pi_{x_{i}}\left(\mathbf{u}_{k}\right) .
$$

In order to discuss the effects of propagation errors introduced to the BP messages, we can consider multiplicative 
error functions, which describe the difference between a true message $m_{j i}\left(\mathbf{x}_{i}\right)$ and its estimation i.e.,

$$
\widehat{m}_{j i}\left(\mathbf{x}_{i}\right)=m_{j i}\left(\mathbf{x}_{i}\right) \cdot e_{j i}\left(\mathbf{x}_{i}\right)
$$

where $m_{j i}$ represents the message from $j$-th to the $i$-th node, and $e_{j i}$ is the error function associated to the message $m_{j i}$. Then, for the message error propagating from node $j$ to node $i$, we can apply a particular functional measure $d\left(e_{j i}\right)$ defined as:

$$
d\left(e_{j i}\right)=\max _{\mathbf{x}_{i}, \mathbf{x}_{j}} \sqrt{\frac{e_{j i}\left(\mathbf{x}_{i}\right)}{e_{j i}\left(\mathbf{x}_{j}\right)}},
$$

where $e$ is the error function related to the information $\mathbf{x}_{i}$ and $\mathbf{x}_{j}$ received from $i$-th and $j$-th node, respectively. Then, we have that $m_{j i}(\mathbf{x})=\hat{m}_{j i}(\mathbf{x}) \forall \mathbf{x}$, if and only if, $\log d\left(e_{j i}\right)=0$.

To facilitate our analysis, we can split (13) into two parts i.e., (i) message products, and (ii) message convolution. In the first part, we obtain:

$$
M_{j i}\left(\mathbf{x}_{j}\right) \propto \psi_{j}\left(\mathbf{x}_{j}\right) \prod_{u \in \Gamma_{j} \backslash i} m_{u j}\left(\mathbf{x}_{j}\right),
$$

where as usual, the proportionality constant is chosen to normalize $M$. We show the message error metric is additive, i.e. that the errors in each incoming message add in their impact on $M$. The second operation is the message convolution, and we obtain:

$$
m_{j i}\left(\mathbf{x}_{i}\right) \propto \int \psi_{j i}\left(\mathbf{x}_{j}, \mathbf{x}_{i}\right) \psi_{j}\left(\mathbf{x}_{j}\right) M_{j i}\left(\mathbf{x}_{j}\right) d \mathbf{x}_{j},
$$

where $M$ is a normalized message or product of messages. Notice that the $\log$ of $d\left(e_{j i}\right)$ is additive, since for several incoming messages $\hat{m}_{u j}$ we have:

$$
\log d\left(\frac{\hat{M}_{j i}}{M_{j i}}\right)=\log d\left(\prod e_{u j}\right) \leq \sum \log d\left(e_{u j}\right) .
$$

Finally, we can derive a minimum rate of contraction on the errors. Let us consider the message from $j$-th to $i$-th node. The error measure $d\left(e_{j i}\right)$ is given by

$$
d\left(e_{j i}\right)^{2}=d\left(\frac{\hat{m}_{j i}}{m_{j i}}\right)^{2} .
$$

subject to certain constraints, such as positivity of the messages and potentials. From (24), we obtain two bounds:

$$
d\left(e_{j i}\right)^{2} \leq d\left(E_{j i}\right)^{2}, \quad d\left(e_{j i}\right)^{2} \leq d\left(\psi_{j i}\right)^{4} .
$$

\section{CONCLUSIONS}

In this paper, we addressed an heterogenous IoT networks scenario, with a plethora of devices for sensing applications. The issue of data sharing and message correction in a multihop IoT environment has been investigated through a Bayesian approach. Specifically, a BP algorithm for message correction, and information update has been presented in its infancy. Future works will address the assessment of the proposed algorithm in an extended simulated scenario.

\section{ACKNOWLEDGMENT}

This work has been partially supported by DAHMS project.

\section{REFERENCES}

[1] S.A. Alvi, G.A. Shah, and W. Mahmood. Energy efficient green routing protocol for internet of multimedia things. In Intelligent Sensors, Sensor Networks and Information Processing (ISSNIP), 2015 IEEE Tenth International Conference on, pages 1-6, April 2015.

[2] Luigi Atzori, Antonio Iera, and Giacomo Morabito. The internet of things: A survey. Comput. Netw., 54(15):2787-2805, October 2010.

[3] F.H. Bijarbooneh, W. Du, E.C.-H. Ngai, X. Fu, and J. Liu. Cloudassisted data fusion and sensor selection for internet-of-things. Internet of Things Journal, IEEE, PP(99):1-1, 2015.

[4] D. Bonino, M.T.D. Alizo, A. Alapetite, T. Gilbert, M. Axling, H. Udsen, J.A.C. Soto, and M. Spirito. Almanac: Internet of things for smart cities. In Future Internet of Things and Cloud (FiCloud), 2015 3rd International Conference on, pages 309-316, Aug 2015.

[5] C. Cervantes, D. Poplade, M. Nogueira, and A. Santos. Detection of sinkhole attacks for supporting secure routing on 6lowpan for internet of things. In Integrated Network Management (IM), 2015 IFIP/IEEE International Symposium on, pages 606-611, May 2015.

[6] R. Giuliano, F. Mazzenga, A. Neri, and A.M. Vegni. Security access protocols in iot networks with heterogenous non-ip terminals. In Distributed Computing in Sensor Systems (DCOSS), 2014 IEEE International Conference on, pages 257-262, May 2014.

[7] Fang Hu, Dan Xie, and Shaowu Shen. On the application of the internet of things in the field of medical and health care. In Green Computing and Communications (GreenCom), 2013 IEEE and Internet of Things (iThings/CPSCom), IEEE International Conference on and IEEE Cyber, Physical and Social Computing, pages 2053-2058, Aug 2013.

[8] Jiong Jin, J. Gubbi, Tie Luo, and M. Palaniswami. Network architecture and qos issues in the internet of things for a smart city. In Communications and Information Technologies (ISCIT), 2012 International Symposium on, pages 956-961, Oct 2012.

[9] Jiong Jin, J. Gubbi, S. Marusic, and M. Palaniswami. An information framework for creating a smart city through internet of things. Internet of Things Journal, IEEE, 1(2):112-121, April 2014.

[10] N. Kumar, N. Chilamkurti, and S. Misra. Bayesian coalition game for the internet of things: an ambient intelligence-based evaluation. Communications Magazine, IEEE, 53(1):48-55, January 2015.

[11] Songtai Li, Xiuquan Qiao, and Xiaofeng Li. Study on the architecture of platform for internet of things service based on edsoa. In Computer and Information Technology (CIT), 2012 IEEE 12th International Conference on, pages 953-959, Oct 2012.

[12] Bin Liu, Zhenfeng Xu, Junjie Chen, and Geng Yang. Toward reliable data analysis for internet of things by bayesian dynamic modeling and computation. In Signal and Information Processing (ChinaSIP), 2015 IEEE China Summit and International Conference on, pages 10271031, July 2015.

[13] V. Miori and D. Russo. Anticipating health hazards through an ontology-based, iot domotic environment. In Innovative Mobile and Internet Services in Ubiquitous Computing (IMIS), 2012 Sixth International Conference on, pages 745-750, July 2012.

[14] Zhijing Qin, G. Denker, C. Giannelli, P. Bellavista, and N. Venkatasubramanian. A software defined networking architecture for the internet-of-things. In Network Operations and Management Symposium (NOMS), 2014 IEEE, pages 1-9, May 2014.

[15] R. Tnjes, E.S. Reetz, K. Moessner, and P.M. Barnaghi. A test-driven approach for life cycle management of internet of things enabled services. In Future Network Mobile Summit (FutureNetw), 2012, pages $1-8$, July 2012.

[16] Xu Xingmei, Zhou Jing, and Wang He. Research on the basic characteristics, the key technologies, the network architecture and security problems of the internet of things. In Computer Science and Network Technology (ICCSNT), 2013 3rd International Conference on, pages 825-828, Oct 2013. 